\title{
Recent advance and challenge of disease-related detection based immunosensor by using antibodies
}

\begin{abstract}
Over the past decades, numerous biosensors have been developed for detection of various diseases. These biosensors used specific biomarkers for their features such as sensitivity, selectivity, low cost and rapid response. This paper gives an overview of the trends in disease-related detection with affinity biosensors. Also, the advances and the challenges of using affinity biosensors are discussed
\end{abstract}

Keywords: antibody, immunosensor, detection

\author{
Volume 4 Issue 3 - 2018 \\ Ongun Mehmet SAKA \\ Department of Pharmaceutical Technology, Ankara University, \\ Turkey
}

Correspondence: Ongun Mehmet SAKA, Associate Professor, Faculty of Pharmacy, Department of Pharmaceutical Technology, Ankara University, Ankara, Turkey, Tel +90 312 2033161, Email omsaka@gmail.com

Received: April 06, 2018 | Published: June 04, 2018

\section{Introduction}

A sensor is a device used to gather information recorded by chemical, physical or biological changes, and then convert the collected information into a qualitative or quantitative signal. Biological sensors (biosensors) consist of a biological sensing element and an electrochemical transducer. Target analyte reacts selectively with recognition elements such as antibodies, enzymes, nucleic acids, and then an electrical signal is produced by transducer. After separation and amplification processes, electrical signal converted into a measurable value. Electrochemical biosensors have been widely developed in last decades. Biomarkers are rationally used as a characteristic indicator of some pathogenic and pharmacological response to therapeutic intervention. One important class of biomarkers includes proteinic molecules that, when present at elevated or depressed concentrations in serum, tissue, or saliva, can be indicative of disease states. Various forms of voltammetry (linear sweep, differential pulse, square-wave,stripping) and amperometry are the most widely used electrochemical methods for detection of disease-related biomarkers. Although some of them are produced commercially and routinely used in clinical, environmental, and industrial analysis. But sometimes, enzyme of the substation or analyte in living organisms couldn't be available to detect, or electrochemical biosystem is too expensive to use. ${ }^{1}$ So that affinity sensors were developed as an acceptable alternative. Affinity biosensors are based on selective interaction between the targeted analyte and biological component such as an antibody (immunosensor) or nucleic acids, DNA/microRNA (electrochemical DNA hybridization biosensor). ${ }^{2}$

The principal of an electrochemical immunosensor simply based on a sandwich immunoreaction that includes a tracer antibody, a capture antibody, and the antigen. ${ }^{3}$ The biomarker is a characteristic indicator of some pathogenic diseases, in biological environment the measurement of tumorigenic antigen markers, such as carcinoembryonic antigen, ${ }^{4}$ prostate-specific antigen, ${ }^{5}$ human-chronic gonadotropin, ${ }^{3}$ can be utilized to screen for some type of cancer disease. ${ }^{5,6}$ Electrochemistrybased approaches for cancer diagnosis with low cost, high sensitivity ${ }^{7}$ were automated with multiplexed protein based electrochemical immunosensors. Kadimisetty et al., ${ }^{8}$ proposed automated multiplexed electrochemiluminescent immunoarrays for cancer biomarker proteins. ${ }^{8}$ Celiac disease, which is known as an autoimmune disorder that primarily affects the gastrointestinal tract, triggers the production of antibodies against gliadin and tissue transglutaminase (tTG). ${ }^{3}$ The golden standard for detection of celiac disease is a biopsy from smallintestine. Researchers improved an electrochemical immunosensor that can be applied to detect tTG antibodies. ${ }^{3,9,10}$ Dulay et al., ${ }^{3}$ indicates that "serological screening tests (eg., enzyme linked immunosorbent assay, ELISA) detects the disease associated autoantibodies have become increasingly more specific and sensitive and it is expected that the official criteria for celiac disease will soon be changed to be a combination of genetic and serology testing". ${ }^{3}$ To provide more specificity and sensitivity serological tests were connected with anti-gliadin and anti-tTG antibodies. ${ }^{9}$ An electrochemical immunosensing strategy was improved to detect antibodies of tTG from human serum with high sensitivity for celiac disease. ${ }^{10}$ Hepatitis $B$ virus primarily replicates in the liver, but also replicates in other organs and lymphocytes. ${ }^{11-13}$ Patients with hepatitis, produces Ig-G antibodies against to hepatitis-B surface antigen (envelope viralprotein). ${ }^{14}$ These antibodies from human sera were used to detect by electrochemical immunosenseors. ${ }^{12,13}$ Alizadeh et al., ${ }^{12}$ produced an affinity immunosensor by using magnetic $\mathrm{Fe}_{3} \mathrm{O}_{4}$ nanoparticles, methylene blue and gold nanoparticles for detection hepatitis- $\mathrm{B}$ surface antigen. ${ }^{12}$

Carcinoembryonic antigen (CEA), which was considered as an oncofetal antigen expressed at cancer patients, was used for detection with $\mathrm{Ag} / \mathrm{Au}$ nanoparticles. ${ }^{4}$ Researchers indicate that CEA immunosensor provides high current response, but the optimal incubation time of the analysis is a critical factor for detection. Tang et al., ${ }^{15}$ detect CEA antibody by fixing covalently with glutathione, ${ }^{15}$ successfully from human serum. The potential of this method is simple and shows efficient strategy for immunoassays. On the other hand it only focused on determination CEA antigen molecules. Researchers improved this method by using CEA/colloid/Au/chitosan with screenprinted carbon electrode ${ }^{16}$ or horseradish peroxide-labelled CEA antibody. ${ }^{15} \mathrm{~A}$ high performance three-dimensional electrochemical immunosensor was developed for sensitive detection of CEA from serum samples by Liu et al., ${ }^{17}$ According to their results, this immunosensor presents excellent performance in terms of detection rate, high stability, and high sensitivity at low detection limit. Dengue disease is caused by mosquitoes infected with RNA-containing 
dengue virus. Dengue disease is one of the most important emerging infectious pathologies. ${ }^{18}$ Molecular binding between virus and its receptor CLEC5A is weak, so that Tung et al., ${ }^{19}$ developed a new method by adapting gold nanoparticles. ${ }^{19}$ This provides the biosensor highly promising tool for screening the actual binding site of antigenglycol conjugation. Ni et al.,${ }^{20}$ developed immunosensor by indicating folate receptors which was over expressed in cancer cells.

\section{Conclusion}

Over the last few years, researchers working on biosensors have widened their interest towards new fields of application. Especially antibodies and other biomolecules have been widely used for accurate detection of hepatitis B, Dengue disease and different type of cancer. Researchers developed electrochemical immunosensors to be simple, sensitive, selective and rapid with a promising potential as a biosensor analyte. Future work is to study whether immunosensors can be extended to detect of other antigens by changing the respective antibodies.

\section{Acknowledgements}

None.

\section{Conflict of interest}

Author declares that there is no conflict of interest.

\section{References}

1. Ronkainen NJ, Halsall HB, Heineman WR. Electrochemical biosensors. Chem Soc Rev 2010;39:1747-1763.

2. Beltran AP, Garcia M. DNA Biosensors and Biomarkers to Cancer Detection. Int J Biosensors\&Bioelectronics. 4(1):00090.

3. Dulay S, Lozano P, Iwuoha E, et al. Electrochemical detection of celiac disease-related anti-tissue transglutaminase antibodies using thiol based surface chemistry. Biosens Bioelectron. 2011;26:3852-3856.

4. Huang J, Tian J, Zhao Y, et al. Ag/Au nanoparticles coated graphene electrochemical sensor for ultrasensitive analysis of carcinoembryonic antigen in clinical immunoassay. Sens Actuators B Chem. 2015;206:570576 .

5. Healy DA, Hayes CJ, Leonard P, et al. Biosensor developments: Application to prostate-specific antigen detection. Trends Biotechnol. 2007;25:125-131.

6. Alizadeh N, Hallaj R, Salimi A. A highly sensitive electrochemical immunosensor for hepatitis B virus surface antigen detection based on Hemin/G-quadruplex horseradish peroxidase-mimicking DNAzymesignal amplification. Biosens Bioelectron. 2017;94:184-192.
7. Dixit CK, Kadimisetty K, Otieno BA, et al. Electrochemistry-based Approaches to Low Cost, High Sensitivity, Automated, Multiplexed Protein Immunoassays for Cancer Diagnostics. Analyst. 2016;141:536547.

8. Kadimisetty K, Malla S, Sardesai N, et al. Automated Multiplexed ECL Immunoarrays for Cancer Biomarker Proteins. Anal Chem. 2015;87:4472-4478.

9. Dahlbom I, Agardh D, Hansson T. Protein A and protein G ELISA for the detection of $\mathrm{IgG}$ autoantibodies against tissue transglutaminase in childhood celiac disease. Clin Chim Acta. 2008;395:72-76.

10. Pividori MI, Lermo A, Bonanni A, et al. Electrochemical immunosensor for the diagnosis of celiac disease. Anal Biochem. 2009;388:229-234.

11. Luo Y, Lo CM, Cheung CK, et al. Identification of hepatitis B virusspecific lymphocytes in human liver grafts from HBV-immune donors. Liver Transplant. 2007;13:71-79.

12. Alizadeh N, Hallaj R, Salimi A. A highly sensitive electrochemical immunosensor for hepatitis B virus surface antigen detection based on Hemin/G-quadruplex horseradish peroxidase-mimicking DNAzymesignal amplification. Biosens Bioelectron. 2017;94:184-192.

13. Reverte L, Prieto-Simon B, Campas M. New advances in electrochemical biosensors for the detection of toxins: Nanomaterials, magnetic beads and microfluidics systems. Anal Chim Acta. 206;908:8-21.

14. Ozbilgin ND, Saka OM, Bozkir A. Preparation and in vitro/in vivo evaluation of mucosal adjuvant in situ forming gels with diphtheria toxoid. Drug Delivery. 2014;21(2):140-147.

15. Tang D, Ren J. In situ amplified electrochemical immunoassay for carcinoembryonic antigen using horseradish peroxidase-encapsulated nanogold hollow microspheres as labels. Anal Chem. 2008;80:8064.

16. Wu J, Tang J, Dai Z, et al. A disposable electrochemical immunosensor for flow injection immunoassay of carcinoembryonic antigen. Biosens Bioelectron. 2006;22:102-108.

17. Liu J, Wang J, Wang T, et al. Three-dimensional electrochemical immunosensor for sensitive detection of carcinoembryonic antigen based on monolithic and macroporous graphene foam. Biosens Bioelectron. 2015;65:281-286.

18. Teles FSRR. Biosensors and rapid diagnostic tests on the frontier between analytical and clinical chemistry for biomolecular diagnosis of dengue disease. Anal Chim Acta. 2011;687:28-42.

19. Tung YT, Wu MF, Wang GJ, et al. Nanostructured electrochemical biosensor for th0065 detection of the weak binding between the dengue virus and the CLEC5A receptor. Nanomed Nanotechnol Biol Med. 2014;10:1335-1341.

20. Ni J, Wang Q, Yang W, et al. Immobilization free electrochemical biosensor for folate receptor in cancer cells based on terminal protection. Biosens Bioelectron. 2016;86:496-501. 